\title{
Iatrogenic Comorbidity in Childhood and Adolescence: New Insights from the Use of Antidepressant Drugs
}

\author{
Emanuela Offidani - Giovanni A. Fava • \\ Nicoletta Sonino
}

Published online: 1 July 2014

(c) Springer International Publishing Switzerland 2014

\begin{abstract}
The term "iatrogenic comorbidity" refers to unfavorable modifications in the course of an illness, with regard to its characteristics and responsiveness, which may be related to previous treatments. Some iatrogenic adverse events arising from either pharmacotherapy or psychotherapy cannot be subsumed under the traditional rubric of adverse effects and require careful evaluation. Children and adolescents are generally more likely to experience adverse health consequences after drug treatment. The use of antidepressant drugs in this age group may cause potential long-term detrimental effects, such as mood elevation that does not subside when drugs are discontinued and may predispose to the development of a bipolar disorder. The concept of iatrogenic comorbidity in children and adolescents has heuristic value in weighing potential benefits and risks associated particularly with psychotropic treatments.
\end{abstract}

E. Offidani

Center for Complementary and Integrative Medicine, Weill

Cornell Medical College, New York, NY, USA

G. A. Fava ( $\square)$

Affective Disorders Program, Department of Psychology, University of Bologna, Viale Berti Pichat 5,

40127 Bologna, Italy

e-mail: giovanniandrea.fava@unibo.it

G. A. Fava $\cdot$ N. Sonino

Department of Psychiatry, State University of New York at Buffalo, Buffalo, NY, USA

N. Sonino

Department of Statistical Sciences, University of Padova, Padua, Italy

\section{Key Points}

Children and adolescents are more likely to experience adverse events of psychotropic drugs

When antidepressant agents are used, adverse reactions are expected to subside upon drug discontinuation but may persist conferring a specific vulnerability that may also affect the response to future treatments

The concept of iatrogenic comorbidity may increase the understanding of adverse phenomena that last after drug discontinuation and may help clinicians in weighing the potential benefits and risks in using any treatment in children and adolescents

\section{Introduction}

There has been growing concern about the adverse health consequences of exposure to environmental agents in children. At least in some instances, such effects tend to be more pronounced in youths than in adults [1]. Even though children and adolescents may present with a better ability to recover, when the exposure to the toxic agent is prolonged or exceeds the threshold of tolerability it may result in adverse effects that are more severe than those taking place in adulthood [1]. Accordingly, psychotropic drugs may exert specific adverse effects according to age, and children and adolescents may display a greater likelihood of treatment-emergent adverse events [2]. Adverse events primarily include altered growth velocity, rash, dystonia, 
tics, affect lability, activation, metabolic blood test abnormalities, sedation, sialorrhea, and electrocardiogram irregularities [3]. Variations in terms of treatment response may be ascribed to the incomplete development of the central nervous system [4]. In particular, a major role has been attributed to the immature adrenergic system in characterizing response to psychotropic drugs in children and young adolescents [4]. Unfortunately, there is little consensus as to the assessment of adverse events in children and adolescents. A review of 196 psychopharmacology articles published between 1980 and 2000 in the pediatric field showed that assessment methods aimed to detect adverse reactions were heterogeneous across studies [5]. An issue that requires further clarification is whether adverse events do always subside upon discontinuation of the drug or may persist, conferring a specific vulnerability and affecting the response to subsequent treatments.

\section{The Concept of Iatrogenic Comorbidity}

\subsection{Behavioral Toxicity of Psychotropic Drugs}

In 1968, Di Mascio and Shader introduced the concept of behavioral toxicity of psychotropic drugs. Such a concept referred to the pharmacologic actions of a drug that, within the dose range in which it has been found to possess clinical utility, may produce alterations in mood, perceptual, cognitive, and psychomotor functions, that limit the capacity of the individual or constitute a hazard to his/her well-being [6-8]. Examples from the use of benzodiazepines and antidepressant drugs in adults were increased anxiety, depression, and rage, as well as euphoria [6-8]. The authors noted that a drug effect such as sedation or motor stimulation may be considered adverse for one patient, and yet therapeutic and desired for another patient; within the same patient it may be of value at one stage of his/her illness and adverse at a later stage. Their formulation received, however, scanty attention in the literature. In 1980, Perl et al. [9] reviewed the concept of behavioral toxicity discussing the mechanisms by which psychotropic drugs can induce adverse reactions. They illustrated that psychotropic drugs can cause behavioral toxicity through the extension of their primary therapeutic action and/or the onset of secondary actions as well as withdrawal, dependence, and tolerance symptoms [9]. Behavioral toxicity may be characterized by oversedation, depression, dystonia, and akathisia with antipsychotic agents; impaired psychomotor and cognitive function, sedation, disinhibition, and confusional states with benzodiazepines; fatigue, somnolence, restlessness, and agitation with antidepressant drugs; and cognitive impairments with lithium [9]. All psychotropic drugs were found to display withdrawal reactions and, in rare cases, paradoxical effects with a particular incidence in older adults and children [9]. A few years later, Van Putten and Marder [10] described akinesia within the behavioral toxicity of antipsychotic drugs.

More recently, Zito et al. [11] underscored that the manifestations of behavioral toxicity have been extensively described with pharmacologic therapies in any medical condition. Yet, they still represent a major challenge in psychiatry, not only because there are major difficulties in distinguishing symptoms of the disease and those induced by treatment, but also because information available in clinical trials are scant and inadequate [11].

\subsection{Iatrogenic Comorbidity}

The concept of behavioral toxicity does not provide differentiation between adverse events that are limited to the period of psychotropic drug administration and effects that may persist long after their discontinuation $[12,13]$. These latter phenomena led to the introduction of the concept of iatrogenic comorbidity in adult psychiatry [12, 13]. Any type of psychotropic drug treatment, particularly after longterm use, may increase the risk of experiencing additional psychopathologic problems (that do not necessarily subside with discontinuation of the drug) or of modifying responsiveness to subsequent treatments $[12,13]$. Examples that are concerned with the use of antidepressant drugs include switching to a bipolar course in patients with unipolar depression, persistent post-withdrawal symptoms after discontinuation of a selective serotonin reuptake inhibitor (SSRI), and resistance to a drug that was previously effective [14]. Negative effects may occur also as a result of psychotherapeutic treatment, whether because of techniques, patient or therapist variables, or inappropriate use [15]. These events that affect both pharmacotherapy and psychotherapy cannot be subsumed under the traditional rubric of adverse effects, but constitute iatrogenic adverse events that require a careful evaluation. Alvan Feinstein's classic definition of comorbidity as "any distinct additional clinical entity that has existed or that may occur during the clinical course of a patient who has the index disease under study" referred also to antecedent pathologic events that were judged to affect the current disease process [16]. The cross-sectional nature of the classification systems in psychiatry has limited the use of the term "comorbidity" to what a patient may be currently experiencing. The term "iatrogenic comorbidity" refers to unfavorable modifications in the course, characteristics, and responsiveness of an illness that may be related to treatments administered previously. Such potential connection should be kept in mind when selecting a new therapeutic approach. 
3 Iatrogenic Comorbidity in Pediatric Patients Treated with Antidepressant Drugs

Iatrogenic comorbidity may have particular relevance to children and adolescents. We will focus on antidepressant drugs, but similar considerations may apply to other areas of psychopharmacology.

\subsection{Use of Antidepressant Drugs in Children and Adolescents}

Even though the use of antidepressant drugs has been advocated in children and adolescents with a number of psychiatric conditions (major depressive disorder, obsessive-compulsive illness, generalized anxiety, and social phobia), the efficacy of antidepressant drugs in the pediatric age is rather controversial. As Leckman [17] emphasized, meta-analyses conducted in pediatric patients treated with SSRIs for depressive or anxiety disorders disclosed very unsatisfactory results. In particular, during SSRI trials, children below 12 years of age with depressive disorders did not differ in response rates from placebo controls, and pediatric patients with anxiety disorders tended to present residual symptoms after reporting an initial improvement.

Bridge et al. [18] conducted a meta-analysis of pediatric trials to assess the balance between clinical benefits and suicide risk in relation to use of antidepressants for major depressive disorders and anxiety disturbances. Antidepressant medications showed greater efficacy over placebo, but also an increased risk of suicidal ideations and suicide attempts across all trials and indications. Whittington and colleagues [19], analyzing data from published and unpublished randomized controlled trials, evaluated the effects of SSRIs versus placebo in children and adolescents. They reported that while published data suggested a favorable risk-benefit profile for some SSRIs, once unpublished data were added, the risks would outweigh the benefits of these drugs in the treatment of depression [19].

\subsection{Manifestations of Iatrogenic Comorbidity Induced by Antidepressant Drugs}

In young patients, antidepressant drugs have been found to induce excessive mood elevation. Symptoms of behavioral activation include irritability, hyperactivity, and sleep problems (insomnia), which often do not subside when drugs are discontinued and may induce the development of a bipolar disorder [20]. A systematic review and metaanalysis of children and adolescent patients disclosed that rates of excessive arousal-activation during treatment with antidepressants were very high both in anxiety $(13.8 \%)$ and depression (9.8\%), compared with placebos (5.2 and
$1.1 \%$, respectively) [20]. Furthermore, during treatment with antidepressant drugs, the incidence of manic or hypomanic manifestations was much higher than that in comparable reports involving anxious adults and similar to that found among depressed adults [20].

Martin and colleagues investigated the risk of manic conversion among more than 87,000 mental health users aged between 5 and 29 years [21]. Results showed that overall the prevalence rate of conversion was $5.4 \%$ $(N=4,748)$. Conversion was found to be threefold more likely in individuals using antidepressants than in those not taking psychotropic medications. These data should be interpreted with caution and need appropriate replication, but may constitute an alarming example of iatrogenic comorbidity in children and adolescents. The child or adolescent may be more liable to develop behavioral activation when subsequent treatments are applied, particularly of a pharmacologic nature, or if he/she seeks the use of stimulants for recreational purposes. The short-term benefits of a treatment should thus be balanced against the potential long-term consequences that cannot be foreseen.

A meta-analysis from the Cochrane Database comparing antidepressant drugs, psychological therapies, and their combination (pharmacotherapy and psychotherapy) in children and adolescents with depression and/or anxiety reported that there was no evidence of superiority of medications over psychological therapy in the longer term [22]. Combination therapy was also not superior to psychological treatment alone. The authors concluded that in the acute phase of the disease, medication exerts a faster response than either placebo or psychological therapies. However, such superiority is not maintained in the long term [22].

\section{Risk, Responsiveness, and Vulnerability Associated with Use of Antidepressant Drugs in Children and Adolescents}

Clinical decisions concerned with the application of knowledge to the individual patient need to be placed in the framework of risk (the likelihood of poor outcomes from an index disorder if therapy is withheld), responsiveness to the treatment option, and vulnerability to the adverse effects of treatment, including those that are subsumed under the rubric of iatrogenic comorbidity [23]. Two clinical cases may illustrate the complexity of events with the use of antidepressant drugs in children and adolescents and the importance of clinical judgment [24].

\subsection{Case 1}

Ann was a 14-year-old girl who developed a school phobia at the beginning of high school. Her parents brought Ann to 
a child psychiatrist who prescribed citalopram $(20 \mathrm{mg}$ per day). After 1 month, she was still refusing to go back to school and the psychiatrist added alprazolam $(0.25 \mathrm{mg}$ twice a day). As one would have expected from the available literature [2], there was no response to this drug combination. Ann's parents thus decided to consult another psychiatrist, who immediately referred her to cognitive behavioral therapy by an experienced psychologist. This psychiatrist, fearing withdrawal problems with both drugs, did not stop the medications, postponing drug tapering and discontinuation to the end of the school year. After the first session of psychotherapy, Ann was back to school, and with a weekly session over 3 months she was able to complete the school year. The psychiatrist then tapered and discontinued alprazolam first, and citalopram later. In both cases, withdrawal symptoms emerged (including suicidal ideation for about 6 weeks after citalopram discontinuation), despite the ongoing psychotherapy. Ann has now completed her second year of high school. She is well and drug free.

\subsection{Case 2}

Thomas was a 15-year-old boy who moved with his family from a northern European country to Italy for a year. What had to be a pleasant change for all the family (and indeed was for his parents and his older sister) became a nightmare for him. Thomas, who experienced social fears and inhibition in his own country, became severely depressed and suicidal. He was evaluated by a psychiatrist in Italy who referred him for cognitive behavior therapy and did not prescribe any medication. However, the treating psychologist after a few sessions detected a worsening of suicidal ideations and impulsivity and asked the psychiatrist to reassess him again. This time, imipramine $25 \mathrm{mg}$ at bedtime was prescribed. In 2 weeks, his mood stabilized and his reactivity to environmental stimuli considerably improved. Thomas started collaborating with homework assignments and completely remitted in 4 months. Imipramine was then discontinued without any problems.

\subsection{Risk, Responsiveness, and Vulnerability}

The first case highlights how the use of antidepressant drugs is inappropriate in most cases of anxiety disorder if a psychotherapeutic alternative is available. Because relapse of anxiety is the rule after stopping the medication, antidepressant drugs cannot provide a permanent solution to the clinical problem. Further, in a recent systematic review, no consistent evidence emerged supporting the advantage of using antidepressant drugs over benzodiazepines in treating anxiety disorders in adults [25]. Indeed, benzodiazepines showed fewer treatment drop-outs and adverse events than antidepressant drugs in panic disorder with and without agoraphobia [26]. A major drive over time in the shift from benzodiazepines to antidepressants in anxiety disorders was the well-known risk of dependence with benzodiazepines [26, 27]. However, in due course after their introduction, similar, if not more pronounced, problems occurred with most of the newer antidepressants [14]. Indeed, withdrawal reactions and post-withdrawal syndromes may ensue, despite slow tapering, with both types of drugs [14]. This was found to occur in the case of Ann. Treatment with citalopram was not beneficial and caused iatrogenic comorbidity (vulnerability to mood changes during the withdrawal phase).

The case of Thomas outlines how in major depressive disorders the decision should be guided by clinical judgment. Imipramine was used only when the patient was found to be refractory to cognitive behavior therapy and for the shortest time possible. In this clinical situation, it was probably life saving and did not involve any iatrogenic comorbidity later on.

\section{Conclusion}

There are several crucial questions for psychopharmacology research in pediatrics. Combination treatment (pharmacotherapy and psychotherapy) is frequently advocated in mood and anxiety disorders of children and adolescents for a faster response. Would a short term advantage justify the risk of long-lasting iatrogenic comorbidity? Additionally, what about treatment of anxiety disorders by antidepressant drugs? If we treat an adolescent with panic disorder with a SSRI and panic attacks wane, how long should drug administration be maintained? Will treatment be still effective 1 year later? How much is it going to cost in terms of iatrogenic comorbidity? How does this intervention compare with a psychotherapeutic approach (particularly cognitive behavior therapy)?

Sensitive methods should be developed for monitoring not only untoward events during the trial, but also potential iatrogenic comorbidity after the trial has terminated. This requires examining a broad range of outcomes over time. Zito and colleagues [11] discussed the considerable limitations of current research methodologies for monitoring psychotropic drug safety. The authors pointed out that the use of pharmacoepidemiologic methods may yield a better comprehension of the relationships between pharmacologic treatments and the onset of behavioral adverse events [11]. Randomized controlled studies should include follow-up phases as a necessary extension of a trial. If a combination of pharmacotherapy and psychotherapy is planned in a treatment arm, the testing of psychotherapy with a pharmacologic placebo appears to be necessary in another 
treatment arm. Identifying subgroups at particular risk of developing iatrogenic comorbidity should also be considered [12]. Another potential area of exploration involves duration of treatment according to stages of illness [28]. According to the model outlined by DiMascio and Shader [6-8], it is conceivable, and yet to be tested, that antidepressant drugs may be very helpful in acute situations, but their disadvantages outweigh their potential benefits in post-acute phases. In the meantime, the concept of iatrogenic comorbidity has heuristic value in weighing potential benefits and risks concerned with treatment choices in children and adolescents. It may apply to any type of psychotropic drug treatment and may refer also to medical adverse effects (e.g., metabolic syndrome after antipsychotic drugs) and potentially to any field of pediatric medicine. For example, evidence from a systematic review of literature showed that subjects aged from 5 to 17 years were more likely than adults to develop weight gain when treated with the second-generation neuroleptic risperidone [29]. Nonpsychiatric drugs may cause behavioral toxicity as well $[30,31]$. Indeed, in general pediatric populations, the concept of iatrogenic comorbidity may be extended to any medical problem that persists after discontinuation of a specific treatment and was not present at the onset of disease.

Acknowledgments Drs. Offidani, Fava, and Sonino have no conflicts of interest to declare. Dr. Offidani's work was supported in part by a grant from Fondazione Cassa di Risparmio di Cesena. No funding was received to prepare this paper.

\section{References}

1. Brent RL, Tanski S, Weitzman M. A pediatric perspective on the unique vulnerability and resilience of the embryo and the child to environmental toxicants. Pediatrics. 2004;113(4 Suppl):935-44.

2. Correll CU, Kratochvil CJ, March JS. Developments in pediatric psychopharmacology: focus on stimulants, antidepressants, and antipsychotics. J Clin Psychiatry. 2011;72(5):655-70.

3. Safer DJ. Age-grouped differences in adverse drug events from psychotropic medication. J Child Adolesc Psychopharmacol. 2011;21:299-309.

4. Bylund DB, Reed AL. Childhood and adolescent depression: why do children and adults respond differently to antidepressant drugs? Neurochem Int. 2007;51(5):246-53.

5. Greenhill LL, Vitiello B, Riddle MA, Fisher P, Shockey E, March JS, Levine J, Fried J, Abikoff H, Zito JM, McCracken JT, Findling RL, Robinson J, Cooper TB, Davies M, Varipatis E, Labellarte MJ, Scahill L, Walkup JT, Capasso L, Rosengarten J. Review of safety assessment methods used in pediatric psychopharmacology. J Am Acad Child Adolesc Psychiatry. 2003;42(6):627-33.

6. Di Mascio A, Shader RI. Behavioral toxicity of psychotropic drugs: I. Definition. II. Toxic effect on psychomotor function. Conn Med. 1968;32:617-20.

7. Di Mascio A, Shader RI. Behavioral toxicity of psychotropic drugs: III. Effect on perceptual and cognitive functions. IV. Effect on emotional (mood) states. Conn Med. 1968;32:771-5.
8. Di Mascio A, Shader RI. Behavioral toxicity of psychotropic drugs: V effects on gross behavioral patterns. Conn Med. 1968; 33:279-81.

9. Perl M, Hall RCW, Gardner ER. Behavioral toxicity of psychiatric drugs. In: Hall RCW, editor. Psychiatric presentations of medical illness. New York: Spectrum Publications; 1980. p. 311-36.

10. Van Putten T, Marder SR. Behavioral toxicity of antipsychotic drugs. J Clin Psychiatry. 1987;48:13-9.

11. Zito MJ, Safer DJ, Craig TJ. Pharmacoepidemiology of psychiatric disorders. In: Hartzema AG, Tilson HH, Chan KA, editors. Pharmacoepidemiology and therapeutic risk management. Cincinnati: Harvey Whitney Books; 2008. p. 817-54.

12. Fava GA, Tomba E, Tossani E. Innovative trends in the design of clinical trials in psychopharmacology and psychotherapy. Progr Neuropsychopharmacol Biol Psychiatry. 2013;40:306-11.

13. Fava GA, Tossani E, Bech P, Berrocal C, Chouinard G, Csillag C, Wittchen HU, Rief W. Emerging clinical trends and perspectives on comorbid patterns of mental disorders in research. Int $\mathbf{J}$ Methods Psychiatr Res. 2014;23(Sl):92-101.

14. Fava GA, Offidani E. The mechanisms of tolerance in antidepressant action. Progr Neuropsychopharmacol Biol Psychiatry. 2011;35(7):1593-602.

15. Barlow DH. Negative effects from psychological treatments. Am Psychol. 2010;65(1):13-20.

16. Feinstein AR. The pre-therapeutic classification of comorbidity in chronic disease. J Chron Dis. 1970;23:455-68.

17. Leckman JF. The risks and benefits of antidepressants to treat pediatric-onset depression and anxiety disorders: a developmental perspective. Psychother Psychosom. 2013;82(3):129-31.

18. Bridge JA, Iyengar S, Salary CB, Barbe RP, Birmaher B, Pincus HA, Ren L, Brent DA. Clinical response and risk for reported suicidal ideation and suicide attempts in pediatric antidepressant treatment: a meta-analysis of randomized controlled trials. JAMA. 2007;297(15):1683-96.

19. Whittington CJ, Kendall T, Fonagy P, Cottrell D, Cotgrove A, Boddington E. Selective serotonin reuptake inhibitors in childhood depression: systematic review of published versus unpublished data. Lancet. 2004;363(9418):1341-5.

20. Offidani E, Fava GA, Tomba E, Baldessarini RJ. Excessive mood elevation and behavioral activation with antidepressant treatment of juvenile depressive and anxiety disorders: a systematic review. Psychother Psychosom. 2013;82(3):132-41.

21. Martin A, Young C, Leckman JF, Mukonoweshuro C, Rosenheck R, Leslie D. Age effects on antidepressant-induced manic conversion. Arch Pediatr Adolesc Med. 2004;158(8):773-80.

22. Cox GR, Callahan P, Churchill R, Hunot V, Merry SN, Parker AG, Hetrick SE. Psychological therapies versus antidepressant medication, alone and in combination for depression in children and adolescents. Cochrane Database Syst Rev. 2012;11: CD008324. doi:10.1002/14651858.CD008324.

23. Richardson WS, Doster LM. Comorbidity and multimorbidity need to be placed in the context of a framework of risk, responsiveness and vulnerability. J Clin Epidemiol. 2014;67: 244-6.

24. Fava GA. Modern psychiatric treatment. Psychother Psychosom. 2013;82:1-7.

25. Offidani E, Guidi J, Tomba E, Fava GA. Efficacy and tolerability of benzodiazepines versus antidepressants in anxiety disorders: a systematic review and meta-analysis. Psychother Psychosom. 2013;82(6):355-62.

26. Balon R. Benzodiazepines revisited. Psychother Psychosom. 2013;82(6):353-4.

27. Rickels K. Should benzodiazepines be replaced by antidepressants in the treatment of anxiety disorders? Fact or fiction? Psychother Psychosom. 2013;82(6):351-2. 
28. Cosci F, Fava GA. Staging of mental disorders: systematic review. Psychother Psychosom. 2013;82(1):20-34.

29. Safer DJ. A comparison of risperidone-induced weight gain across the age span. J Clin Psychopharmacol. 2004;24(4):429-36.

30. Hall RCW, Stickney SK, Gardner ER. Behavioral toxicity of nonpsychiatric drugs. In: Hall RCW, editor. Psychiatric presentations of medical illness. New York: Spectrum Publications; 1980. p. 337-406.

31. Fava GA, Sonino N. Depression associated with medical illness: treatment considerations. CNS Drugs. 1996;5:175-89. 